\title{
Electrochemically inert aluminum cation coordinated with tetrahydroxybenzoquinone towards high-energy storage
}

Guochun Ding a, c, 1, Limin Zhu a, c, 1,*, Qing Han ${ }^{\text {a, c }}$, Lingling Xie ${ }^{\text {b, c }}$, Xinli Yang a, c, Xin $\mathrm{Li}^{\mathrm{a}}{ }^{\mathrm{a}}$, , Lei Chen ${ }^{\mathrm{d}}$, Gongke Wang ${ }^{\mathrm{e}}, \mathrm{Fu}$ Sun ${ }^{\mathrm{f}, *}$, Xiaoyu Cao ${ }^{\text {a, c, } *}$

a School of Chemistry and Chemical Engineering, Henan University of Technology, Zhengzhou 450001, PR China

${ }^{\mathrm{b}}$ School of Environmental Engineering, Henan University of Technology, Zhengzhou 450001, PR China

${ }^{\mathrm{c}}$ Key Laboratory of High Specific Energy Materials for Electrochemical Power Sources of Zhengzhou City, Zhengzhou 450001, PR China

${ }^{\mathrm{d}}$ College of Chemical and Printing-dyeing Engineering, Henan University of Engineering, Zhengzhou 450007, PR China

e School of Materials Science and Engineering, Henan Normal University, Xinxiang 453007, PR China

${ }^{\mathrm{f}}$ Qingdao Institute of Bioenergy and Bioprocess Technology Chinese Academy of Sciences, Qingdao 266101, PR China

* Corresponding author

E-mail address: caoxy@haut.edu.cn (X. Cao), liminzhu@haut.edu.cn (L. Zhu), sunfu1998@gmail.com (F.Sun)

${ }^{1}$ These authors contributed equally.

\section{Experimental section}

Synthesis of PTHBQ-AI and PTPAn 
Before adding stoichiometric mass of $\mathrm{AlCl}_{3}$ and THBQ to deionized water, THBQ was first dissolved in deionized water at a nitrogen atmosphere, forming brown solution. Ten minutes later with vigorous stirring at room temperature, the mixture was added with $60 \mathrm{~mL} \mathrm{NaOH}\left(1 \mathrm{~mol} \mathrm{~L}^{-1}\right)$ solution. The solution instantly turned atropurpureus with precipitate. Finally, the product was filtrated, washed with ethanol, methanol and acetone, and dried in a vacuum at $60^{\circ} \mathrm{C}$.

The synthesis of PTPAn sample was an oxy-polymerization method. In a typical synthesis, triphenylamine (TPA) and $\mathrm{FeCl}_{3}$ powder with a mass ratio of 1: 4 were dissolved in $\mathrm{CHCl}_{3}$ solvent, respectively. The two solution underwent magnetic stirring for 10 minutes in a nitrogen atmosphere. Then, the mixed solution was blended at $50{ }^{\circ} \mathrm{C}$ for 2 days before suction filtration and washing with $\mathrm{H}_{2} \mathrm{O}$ and ethanol for several times. Finally, the PTPAn product was dried at $60^{\circ} \mathrm{C}$ for 24 hours in a vacuum.

\section{Materials characterization}

Crystal characteristics of materials were tested by powder X-ray diffraction (XRD, Rigaku MiniFlex 600) with the resource of monochromatic $\mathrm{Cu} \mathrm{K} \alpha$ radiation $(\lambda=1.54$ $\AA$ ). Coordination bond of $\mathrm{P}(\mathrm{THBQ}-\mathrm{Al})$ was confirmed by X-ray photon electron spectroscopy (XPS, ESCALAB 250X) with the excitation source of Al Ka radiation. The surface morphology and internal structure were observed using scanning electron microscopy (SEM, FEI-Quanta 250 FEG). Thermogravimetric analysis (TGA, Labsys NETZSCH TG-209) was performed at a heating rate of $10{ }^{\circ} \mathrm{C} \mathrm{min}{ }^{-1}$ in $\mathrm{N}_{2}$ atmosphere to analyze the thermal stability of PTHBQ-Al and THBQ.

\section{Electrochemical characterization}


Cathode electrodes included active materials, Ketjen black (KB) and poly (vinylidene fluoride) (PVdF) in NMP with a ratio of 6:2:2 for both PTHBQ-Al and THBQ. The slurry was then casted onto an aluminum foil. CR2016-type coin cells consisting of a $\mathrm{Na}$ metal anode and $1 \mathrm{M} \mathrm{NaClO}_{4}$ in diethyl carbonate (DEC) and ethylene carbonate $(\mathrm{EC})$ electrolyte $(\mathrm{DEC}: \mathrm{EC}=1: 1$, by volume ratio) were elaborated in an argon-filled glove box. For LIBs, the electrolyte is $1 \mathrm{M} \mathrm{LiPF}_{6}$ in diethyl carbonate (DEC) and ethylene carbonate (EC) electrolyte (DEC:EC $=1: 1$, by volume ratio). The anode is Li metal. Galvanostatic discharge/charge tests were performed on a Land battery tester. Cyclic voltammetry (CV) was measured on a $\mathrm{CHI} 660 \mathrm{~d}$ electrochemical workstation. For ex situ FT-IR, the collected electrodes obtained from disassembled coin cells in an argon-filled glovebox were washed with dimethyl carbonate (DMC) to explore their mechanism. Ex situ SEM was used to observe the structural stability of electrodes after cycle to confirm the excellent cyclic stability of polymers. For full cell measurements in LIBs and SIBs, polytriphenylamine (PTPAn) was selected as the cathodes and PTHBQ-Al as anodes. The PTPAn cathodes were made up of $70 \%$ active material, 20\% KB and 10\% polytetrafluroethylene (PTFE 60wt.\%). The PTHBQ-Al anodes were made up of $40 \%$ active material, $50 \% \mathrm{~KB}$ and $10 \%$ PTFE. All the cathodes and anodes were pressed on aluminium mesh and dried in vacuum for $24 \mathrm{~h}$ at $60{ }^{\circ} \mathrm{C}$. Certainly, a slightly excess mass of cathodes was used to maintain the capacity of full cell determined by the cathodes.

\section{Cell assembly, electrochemical cycle and settings for tomography measurement}

The housing of the customized tomography cell, the polyether ether ketone 
(PEEK), was purchased from Kaiwei Co., Ltd. The electrolyte of $1 \mathrm{M} \mathrm{LiPF} 6$ in a volume-ratio mixture (1:1) of ethylene carbonate (EC) and ethyl methyl carbonate (EMC) and NMP (N-Methyl-2-pyrrolidone, 99.5\%) were purchased from Sigma Aldrich. Lithium and Celgard 2325 separator were purchased from MTI Cor. USA. All tomography cells were built with $0.2 \mathrm{~mL}$ electrolyte and the employed separators were $3.0 \mathrm{~mm}$. The diameter of the employed $\mathrm{Li}(\mathrm{Na})$ discs was $2.5 \mathrm{~mm}$. During battery assembly, the separator was sandwiched between the Li $(\mathrm{Na})$ disc and the organic electrode. In LIBs, PTHBQ-Al cell was discharged/charged at $25 \mathrm{~mA} \mathrm{~g}^{-1}$ for 54 cycles and $15 \mathrm{~mA} \mathrm{~g}^{-1}$ for 102 cycles, respectively. In SIBs, PTHBQ-Al cell was discharged/charged at $21 \mathrm{~mA} \mathrm{~g}^{-1}$ for 28 cycles and $27 \mathrm{~mA} \mathrm{~g}^{-1}$ for 56 cycles, respectively. The PTHBQ-Al cell discharged/charged for 102 cycles in LIBs were measured at DESY P05 beamline and other PTHBQ-Al cells were measured at BAMline beamline.

BAMline beamline at BESSY II (Berlin, Germany): The energy of the synchrotron beam was monochromatized to $20 \mathrm{KeV}$ using a double monochromator with an energy resolution of about $1.5 \%$. The detector system comprised a $60 \mu \mathrm{m}$ thick $\mathrm{CdWO}_{4}$ scintillator, a microscopic optic and a pco4000 camera equipped with a $4008 \times 2672$ pixels CCD chip that was kept out of the direct beam by using a mirror. A detector system with $0.438 \mu \mathrm{m}$ pixel was used and the corresponding field of view was $1.7 \times 1.2$ $\mathrm{mm}^{2}$ (length $\times$ height). For tomography measurements measured here, 1400 projections within a $180^{\circ}$ battery rotation were recorded with the exposure time of $0.8 \mathrm{~s}$. Additionally, a 2 by 2 binning was used to further decrease the measurement time because of the limited allocated beam time. The exposure-time decreased and binned 
measurements took $\sim 30$ minutes and the resultant spatial resolution is $0.876 \mu \mathrm{m}$.

P05 beamline at DESY (Hamburg, Germany): For the current measurements, the energy was monochromatized to $20 \mathrm{KeV}$ using a double multilayer monochromator $(\mathrm{DMM})$, which provides an energetical beam $\left(\Delta \mathrm{E} / \mathrm{E} \approx 10^{-2}\right)$ with high flux. $\mathrm{A} \mathrm{CdWO}_{4}$ single crystal scintillator of $100 \mu \mathrm{m}$ thickness converts the X-ray to visible light. A fast KIT CMOS camera that was kept out of the direct beam by using a mirror was employed. The used field of view was $3.28 \times 2.46 \mathrm{~mm}^{2}$ (length $\times$ height). For samples measured here, 2400 projections within a $180^{\circ}$ battery rotation were recorded with the exposure time of $160 \mathrm{~ms}$. Every tomography took $\sim 10$ minutes. The achieved spatial resolution was $\sim 0.6 \mu \mathrm{m}$. It has to be noted that for samples measured here, a binning process of 2 by 2 was used when reconstructing the datasets. As a result, the final spatial resolution is $\sim 1.2 \mu \mathrm{m}$.

The uncycled LIB and NIB cells were measured by Rigaku CT lab HX. These two cells were measured under an automatic mode, i.e., the Continuous mode. The scan mode is High Resolution and the scan time is $68 \mathrm{~min}$. During the measurement, the $\mathrm{X}$ ray tube voltage is $70 \mathrm{kV}$ and the tube current is $116 \mu \mathrm{A}$. The geometry between the sample and the detector is Long and the used field of view (FOV) is 10. The distance between Focus and Object is $32 \mathrm{~mm}$ and the distance between Focus and Detector is $432 \mathrm{~mm}$. The resultant spatial resolution is $3.6 \mu \mathrm{m}$.

Tomography Data Processing: The raw tomography data from BAMline and P05 beamline were processed using in-house reconstruction software programmed in IDL 8.2. The data was first normalized, de-noised and in some cases, filtered and underwent 
phase retrieval. Then the filtered back projection was used for final reconstruction. For the data measured by the Rigaku CT lab HX, they were reconstructed and analyzed by the software Tomoshop. 